\title{
Magnesium ion implantation on a micro/ nanostructured titanium surface promotes its bioactivity and osteogenic differentiation function
}

This article was published in the following Dove Press journal:

International Journal of Nanomedicine

21 May 2014

Number of times this article has been viewed

\author{
Guifang Wang ${ }^{1,2, *}$ \\ Jinhua $\mathrm{Li}^{3, *}$ \\ Wenjie Zhang ${ }^{1,2}$ \\ Lianyi $\mathrm{Xu}^{1,2}$ \\ Hongya Pan² \\ Jin Wen ${ }^{1,2}$ \\ Qianju Wu ${ }^{1,2}$ \\ Wenjun She' \\ Ting Jiao' \\ Xuanyong Liu $^{3}$ \\ Xinquan Jiang ${ }^{1,2}$ \\ 'Department of Prosthodontics, Ninth \\ People's Hospital, School of Medicine, \\ Shanghai Jiao Tong University, ${ }^{2}$ Oral \\ Bioengineering Laboratory, Ninth People's \\ Hospital Affiliated to Shanghai Jiao Tong \\ University, School of Medicine, Shanghai \\ Key Laboratory of Stomatology, ${ }^{3}$ State Key \\ Laboratory of High Performance Ceramics \\ and Superfine Microstructure, Shanghai \\ Institute of Ceramics, Chinese Academy \\ of Sciences, Shanghai, People's Republic \\ of China \\ *These authors contributed equally to this \\ work
}

Correspondence: Xinquan Jiang

Department of Prosthodontics, Ninth

People's Hospital affiliated to Shanghai

Jiao Tong University, School of Medicine,

639 Zhizaoju Road, Shanghai 2000II,

People's Republic of China

Tel +86 2I 6313 54I2

Fax +862163136856

Email xinquanj@aliyun.com

Xuanyong Liu

State Key Laboratory of High

Performance Ceramics and Superfine

Microstructure, Shanghai Institute of

Ceramics, Chinese Academy of Sciences,

Shanghai 200050, People's Republic

of China

Tel +862 2I 524I 2409

Fax +86 2I 524I 2409

Email xyliu@mail.sic.ac.cn
Abstract: As one of the important ions associated with bone osseointegration, magnesium was incorporated into a micro/nanostructured titanium surface using a magnesium plasma immersion ion-implantation method. Hierarchical hybrid micro/nanostructured titanium surfaces followed by magnesium ion implantation for 30 minutes $(\mathrm{Mg} 30)$ and hierarchical hybrid micro/nanostructured titanium surfaces followed by magnesium ion implantation for 60 minutes ( $\mathrm{Mg} 60)$ were used as test groups. The surface morphology, chemical properties, and amount of magnesium ions released were evaluated by field-emission scanning electron microscopy, energy dispersive X-ray spectroscopy, field-emission transmission electron microscopy, and inductively coupled plasma-optical emission spectrometry. Rat bone marrow mesenchymal stem cells (rBMMSCs) were used to evaluate cell responses, including proliferation, spreading, and osteogenic differentiation on the surface of the material or in their medium extraction. Greater increases in the spreading and proliferation ability of rBMMSCs were observed on the surfaces of magnesium-implanted micro/nanostructures compared with the control plates. Furthermore, the osteocalcin $(O C N)$, osteopontin $(O P N)$, and alkaline phosphatase $(A L P)$ genes were upregulated on both surfaces and in their medium extractions. The enhanced cell responses were correlated with increasing concentrations of magnesium ions, indicating that the osteoblastic differentiation of rBMMSCs was stimulated through the magnesium ion function. The magnesium ion-implanted micro/nanostructured titanium surfaces could enhance the proliferation, spreading, and osteogenic differentiation activity of rBMMSCs, suggesting they have potential application in improving bone-titanium integration.

Keywords: surface modification, micro/nanostructure, magnesium, ion implantation, osteogenic differentiation

\section{Introduction}

Titanium has been successfully used in clinical applications due to its excellent biocompatibility, especially in bone anchoring systems such as dental and orthopedic implants and other internal fixation applications. Satisfactory osseointegration must be achieved to ensure that a long and stable functional dental implant is retained in the mandible or long bone. ${ }^{1}$ Since surface characteristics are critical factors influencing interactions between the cell or extracellular matrix and implants that can lead to eventual osseointegration, ${ }^{2}$ various surface treatment methods have been employed to optimize dental implant surfaces for improving their bioactivity and osseointegration. ${ }^{3}$

Surface modification of dental implants involves several features, including their topography, free energy, crystal structure, and chemical composition. ${ }^{4-7}$ Among these, modifications in topography and chemical composition are particularly critical factors for methods of alteration of titanium surfaces. 
For modification of surface topography, it has been suggested that microtopographies can enhance osteoblast differentiation and production of local growth factors in vitro, ${ }^{8,9}$ and promote bone-to-implant contact. ${ }^{10}$ Nanotopographies can directly correspond to the sizes of proteins and membrane receptors that could also increase osteoblast differentiation and tissue regeneration. ${ }^{11}$ Taking into account the importance of both microtopographical and nanotopographical features, the synergistic effects of dual-length scale materials show promise for improving osseointegration. ${ }^{12}$ In a previous study, a hierarchical hybrid micro/nanotip film was fabricated on a titanium surface (NT) using acid etching to form micropits; a sodium titanate nanotip texture was produced using hydrothermal treatment, and $\mathrm{H}^{+}$was then incorporated using an ion exchange process. The optimized NT surfaces showed better bioactivity and osteogenic activity compared with microrough titanium surfaces treated by acid etching alone. ${ }^{13}$

Another significant approach for improving the biological performance of dental and orthopedic implants is alteration in surface chemical characteristics. Incorporation of bioactive trace elements such as calcium, silicon, zinc, strontium, and magnesium is frequently used. ${ }^{14-18}$ These trace elements are able to stimulate bone growth and bone healing by enhancing osteoblast activity. ${ }^{19-21}$ As one of the most abundant elements in the human body, ${ }^{22}$ magnesium is essential for bone metabolism and stimulates new bone formation, and may also interact with integrins of osteoblasts, which are responsible for cell adhesion and stability. ${ }^{23,24}$ Cell responses were enhanced with incorporation of magnesium ions, which appeared to correlate with an increasing concentration of magnesium ions. ${ }^{25}$ For example, Cho et al reported that magnesium ions implanted in resorbable blasting media-treated titanium surfaces using the plasma source ion implantation technique enhanced their capacity for osseointegration. ${ }^{26}$ In another study, implantation of magnesium ions on commercial pure titanium that was treated with sand blasting using large grit and acid etching ${ }^{27}$ improved initial cellular attachment, resulting in enhancement of osteoblast differentiation. Together, these studies indicate the potential of magnesium for improving osseointegration in vivo. Plasma immersion ion implantation stands out from other surface modification processes used to incorporate metal ions into titanium surfaces (eg, plasma spraying, electrolytic processes, sol-gel, and laser cladding) because of its high accuracy in controlling the concentration and depth distribution of impurities. ${ }^{28}$

Hence, we hypothesized that a specific micro/ nanostructured surface NT with good bioactivity and osteogenic activity might possess better bioactive properties and further enhance the osteogenic activity of rat bone marrow mesenchymal stem cells (rBMMSCs) when incorporated with magnesium by plasma immersion ion implantation. We aimed to fabricate and evaluate this micro/nanostructured titanium surface with respect to its potential properties for future applications in osseointegration.

\section{Materials and methods Fabrication and modification of specimens}

Commercial pure titanium (grade 2, $99.5 \mathrm{wt} \%$ purity) plates were ultrasonically cleaned in ethanol and deionized water several times, followed by pickling in a $5 \mathrm{wt} \%$ oxalic acid solution at $100^{\circ} \mathrm{C}$ for 2 hours to remove the oxide layer and obtain a homogeneous surface. The samples were then ultrasonically cleaned in deionized water and dried in an ambient atmosphere for further use. The $\mathrm{TiO}_{2}$ structure on the titanium surface was hydrothermally fabricated according to methods described in our recent work. ${ }^{13}$ Pretreated titanium plates were placed in a Teflon-liner reaction vessel containing an aqueous solution of $7.5 \mathrm{~mL} \mathrm{H}_{2} \mathrm{O}_{2}$ (30 wt \%) and $2.5 \mathrm{~mL} \mathrm{NaOH}(5 \mathrm{M})$ at $80^{\circ} \mathrm{C}$ for 24 hours. Subsequently, the plates were gently rinsed with deionized water and then underwent protonation in $\mathrm{HCl}$ aqueous solution $(0.1 \mathrm{M})$ for 2 hours. Next, the protonated titanium plates were rinsed to neutral with deionized water and dried in an ambient atmosphere, followed by calcination at $450^{\circ} \mathrm{C}$ for one hour to crystallize. This hierarchical micro/NT was then used in the ion implantation process.

Magnesium was implanted into the micro/nanostructured titanium surface using a filtered cathodic arc plasma source for 30 minutes (Mg30) or 60 minutes (Mg60). The instrumental parameters for implantation are listed in Table 1.

\section{Surface characterization}

The surface morphology was characterized using a Magellan 400 field-emission scanning electron microscope (FEI, Hillsboro, OR, USA) equipped with an energy-dispersive

Table I Instrumental parameters used in magnesium plasma immersion ion implantation

\begin{tabular}{lll}
\hline & Target & Cathodic arc \\
\hline Voltage pulse duration $(\mu \mathrm{sec})$ & 450 & 450 \\
Pulsing frequency $(\mathrm{Hz})$ & 7 & 7 \\
lon implantation voltage $(\mathrm{kV})$ & -15 & \\
lon implantation time (minutes) & $30 / 60$ & \\
Pressure $(\mathrm{Pa})$ & $3.6 \times 10^{-3}$ & \\
\hline
\end{tabular}


X-ray spectrometer, which was utilized to determine the chemical composition of the titanium surface. Further, transmission electron microscopic analysis was performed using a JEM-2100F field-emission microscope (JEOL Ltd, Tokyo, Japan), with an accelerating voltage of $200 \mathrm{kV}$. Samples for the investigation were scratched from the substrate and dispersed in ethanol by ultrasound. A droplet of the suspension was placed on a holey copper grid covered with porous carbon film.

\section{Cell culture}

rBMMSCs were isolated from 4-week-old male SpragueDawley rats and cultured according to our previously published procedures. ${ }^{29}$ Briefly, bone marrow was obtained from rat femurs after both ends were cut off at the epiphysis and rinsed using Dulbecco's modified Eagle's medium (DMEM) with 10\% fetal bovine serum and $200 \mathrm{U} / \mathrm{mL}$ heparin. Cells were cultured in DMEM with $10 \%$ fetal bovine serum in an incubator with an atmosphere of 5\% $\mathrm{CO}_{2}$ at $37^{\circ} \mathrm{C}$. Nonadherent cells were removed by the first medium change after 48 hours. Cells at passages 2-3 were used in this study.

\section{Cell morphology and proliferation}

For cell morphology, ${ }^{30}$ cells were seeded on the substrates in a 24 -well plate at a density of $5.0 \times 10^{4}$ cells per $\mathrm{mL}$. The nonadhered cells were removed by washing three times with phosphate-buffered saline after 24 hours of culture. They were fixed in $4 \%$ paraformaldehyde for 30 minutes, and then rinsed with phosphate-buffered saline. These samples were treated with $0.5 \%$ Triton X-100 in phosphate-buffered saline to permeabilize the cells for 20 minutes and then blocked with $1 \%$ bovine serum albumin for 20 minutes. The actin cytoskeletons were labeled by incubating with $100 \mathrm{nM}$ fluorescein isothiocyanate-phalloidin (cytoskeleton) for 60 minutes, and then mounted on glass slides and observed.

The MTT 3-(4,5-dimethylthiazol-2-yl)-2,5diphenyltetrazolium bromide) assay was used to evaluate the proliferation activity of rBMMSCs on different substrates. ${ }^{31}$ After one and 4 days of culture, MTT solution was added to each targeted well, and the specimens were incubated for 4 hours to form formazan. The formazan was dissolved in dimethylsulfoxide and measured at $540 \mathrm{~nm}$ (reference value $630 \mathrm{~nm}$ ) using an ELX ultra microplate reader (BioTek, Winooski, VT, USA). The results are expressed as units of optical density absorbance values. The experiment was performed in triplicate.

\section{Alkaline phosphatase activity}

Cells were seeded on the specimens in 24-well plates at a density of $2 \times 10^{4}$ cells per mL. After 14 days of culture, the cells were fixed and stained using an alkaline phosphatase kit (Shanghai Hongqiao Medical Reagent Company, Shanghai, People's Republic of China). ${ }^{32,33}$ A semiquantitative analysis of alkaline phosphatase was performed as previously described. ${ }^{34}$ Alkaline phosphatase activity was determined by measuring the optical density values for absorbance at $405 \mathrm{~nm}$ after incubation with $p$-nitrophenyl phosphate (Sigma-Aldrich, St Louis, MO, USA) for 30 minutes at $37^{\circ} \mathrm{C}$. The intracellular total protein content was determined using the Micro BCA protein assay kit (Thermo Fisher Scientific, Waltham, MA, USA), and the alkaline phosphatase activity was normalized to the total protein content.

\section{Reverse transcription and real-time polymerase chain reaction assay}

Expression of the osteogenesis-related genes osteocalcin $(O C N)$, osteopontin $(O P N)$, and alkaline phosphatase $(A L P)$ was evaluated using real-time polymerase chain reaction. ${ }^{35,36}$ Cells were seeded with $2 \times 10^{4}$ cells per $\mathrm{mL}$ and cultured for 14 days. Total RNA was isolated using the TRIzol reagent. Equivalent RNA from each sample was reverse-transcribed into complementary DNA using the PrimeScript ${ }^{\mathrm{TM}}$ reverse transcription reagent kit (TaKaRa, Bio Inc, Otsu, Japan). The forward and reverse primers for the selected genes were based on previous research. ${ }^{37}$ Expression levels of these genes were quantified using real-time polymerase chain reaction with SYBR Premix Ex Taq II (TaKaRa). The relative expression level for each gene of interest was normalized to that of the housekeeping gene $G A P D H .{ }^{38}$

\section{Western blot analysis}

Western blot analysis was used to investigate the expression of various osteogenic-related proteins, including $\mathrm{OCN}$, OPN, and alkaline phosphatase, in cells incubated on the tested titanium specimens. The rBMMSCs were seeded on the titanium specimens at a density of $5.0 \times 10^{4}$ cells per $\mathrm{mL}$ for 7 days. Cells were then harvested with a cell protein extraction reagent containing a protease inhibitor cocktail, phosphatase inhibitor cocktail, and phenylmethanesulfonyl fluoride (KangChen Bio-tech Inc., Shanghai, People's Republic of China). Equal amounts of protein were separated by sodium dodecyl sulfate-polyacrylamide gel electrophoresis and transferred onto polyvinylidene difluoride membranes. The blots were blocked with a buffer containing $5 \%$ defatted milk and reacted with primary antibodies. The 
primary antibodies were anti-OCN (Abcam, Cambridge, UK), anti-OPN (Abcam), anti-alkaline phosphatase (Proteintech Europe, Manchester, UK), and anti- $\beta$-actin (Sigma-Aldrich). Membranes were then incubated with horseradish peroxidase-conjugated secondary antibodies including goat anti-rabbit and goat anti-mouse (Beyotime Institute of Biotechnology, Jiangsu, People's Republic of China). Protein bands were visualized using an enhanced chemiluminescence detection system (Alliance; UVItec Limited, Cambridge, UK).

\section{Effects of extracts on rBMMSCs}

The accumulated magnesium ion release from each group of plates was investigated using DMEM. Each sample $\left(1 \mathrm{~cm}^{2}\right)$ was immersed in $1 \mathrm{~mL}$ DMEM for 3 days and then moved to another $1 \mathrm{~mL}$ of fresh DMEM for the next 3 days. The amount of magnesium released in DMEM solution was measured using inductively coupled plasma atomic emission spectrometry (Nu Instruments, Wrexham, UK), with the control specimens (NT) used as blanks.

After 12 days of incubation, the collected extracts were supplemented with $10 \%$ fetal bovine serum and used in cell culture experiments. Cells were seeded in plates at a density of $2.0 \times 10^{4}$ cells per $\mathrm{mL}$. After 4 hours of incubation, DMEM was replaced with different extracts from each type of sample, and then MTT cell metabolic assays, alkaline phosphatase activity assays, and real-time polymerase chain reaction assays were performed as described above.

\section{Statistical analysis}

The data are expressed as the mean \pm standard deviation. The results of assays were compared using analysis of variance with Student-Newman-Keuls post hoc tests by SAS version 8.2 software (SAS Institute, Cary, NC, USA). $P<0.05$ was considered to be statistically significant.

\section{Results}

\section{Sample characterization}

Figure 1 shows the surface morphology of the titanium plates after hydrothermal treatment and subsequent magnesium plasma immersion ion implantation $(\mathrm{Mg}$ PIII) processing. After reacting with alkaline hydrogen peroxide, a homogeneous nanotip structure emerged on the titanium surface, almost perpendicular to the substrate (Figure 1A). The corresponding energy-dispersive X-ray spectrum showed that only titanium and oxide elements were detected, confirming the complete replacement of sodium ions by hydrogen ions (Figure S2). After Mg PIII
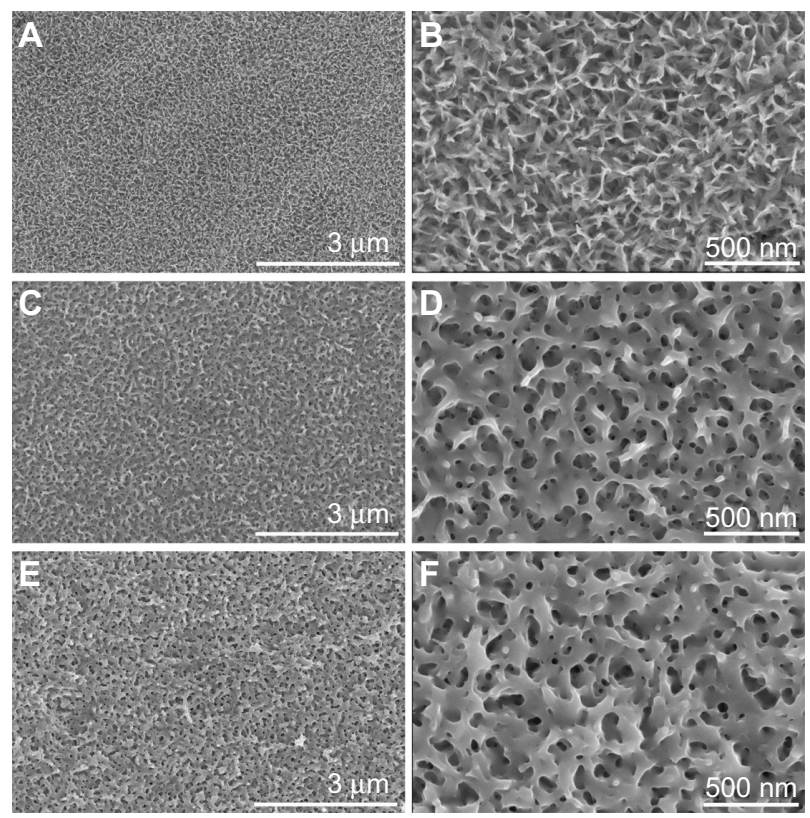

Figure I Surface morphology of three samples at different magnifications. (A and B) NT, (C and D) Mg30, and (E and F) Mg60.

Abbreviations: NT, hierarchical hybrid micro/nanostructured titanium surfaces; $\mathrm{Mg30}$, hierarchical hybrid micro/nanostructured titanium surfaces followed by magnesium ion implantation for 30 minutes; Mg60, hierarchical hybrid micro/ nanostructured titanium surfaces followed by magnesium ion implantation for 60 minutes.

processing for 30 minutes, the nanotip topography was altered from the visual field of scanning electron microscopy at high magnification, as shown in Figure 1D. The corresponding energy-dispersive X-ray spectrometric mapping of magnesium, titanium, and oxygen elements demonstrated the uniform distribution of magnesium on the sample surface (Figure S1). Prolonging the PIII time to 60 minutes did not significantly change the surface topography (Figure 1E and F).

The Mg30 and Mg60 samples underwent further exploration using transmission electron microscopy techniques. Figure 2A shows the typical bright-field transmission electron microscopic image of the sample scratched from the Mg30 surface. The corresponding energy-dispersive $\mathrm{X}$-ray spectrum was also acquired, demonstrating the existence of magnesium, titanium, and oxygen elements, with a magnesium loading content of $2.70 \%$ (Figure 2B). After Mg PIII for 60 minutes, the nanotopography of Mg60 resembled that of $\mathrm{Mg} 30$ (Figure 2C), with an increased magnesium loading content of $4.50 \%$ (Figure 2D). During transmission electron microscopy measurement, the testing samples were powders scratched from the modified titanium surface, which minimized the influence of the metallic titanium substrate and provided an accurate estimation of magnesium content. 

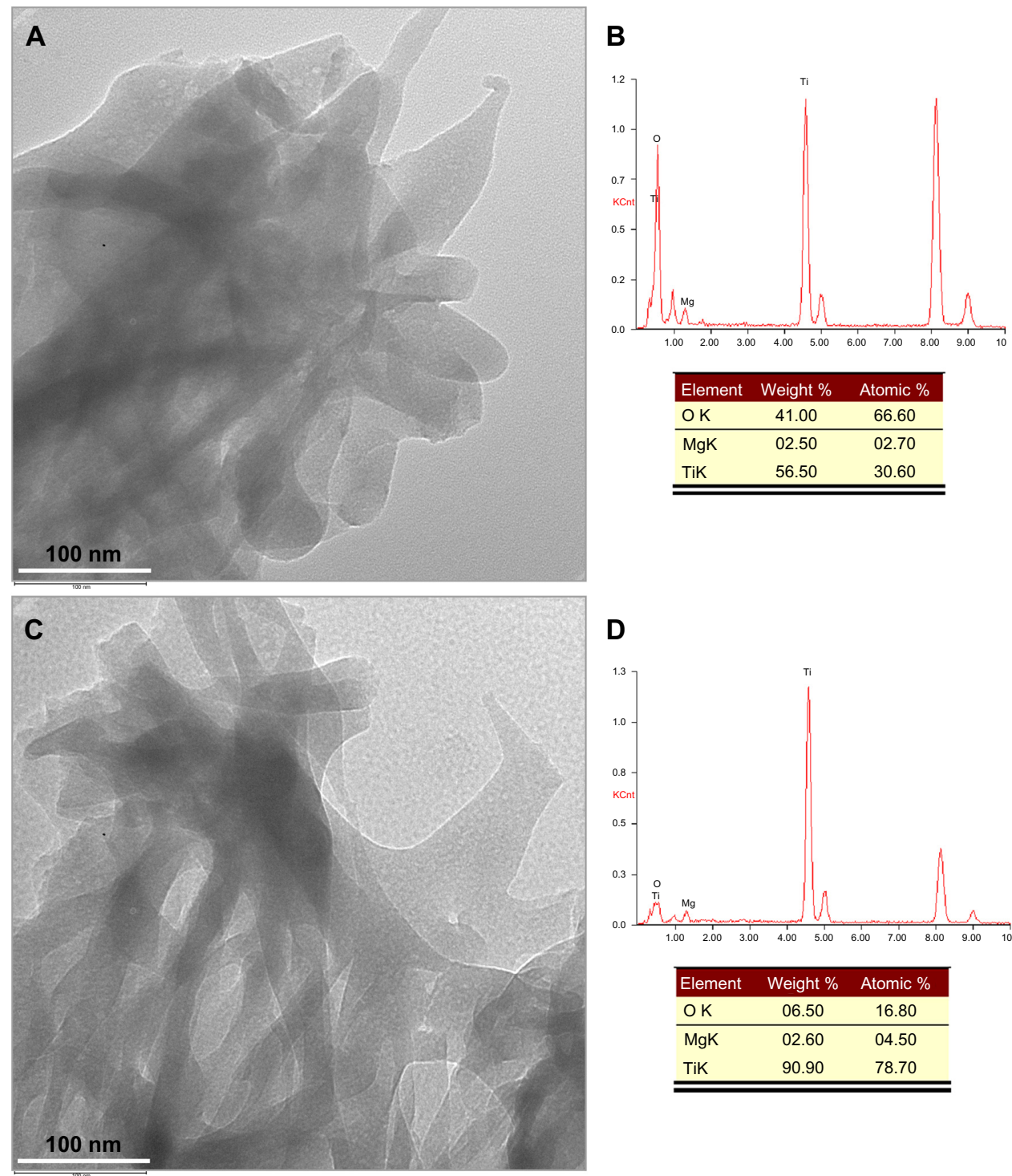

\section{D}

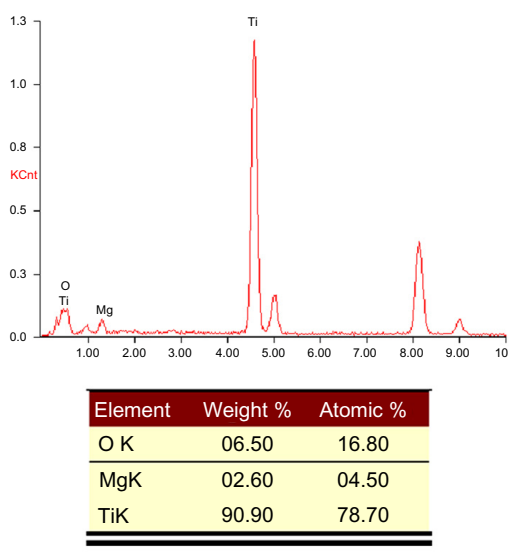

Figure 2 Transmission electron microscopic analysis of two samples incorporating magnesium. (A and B) Mg30 and (C and D) Mg60.

Abbreviations: $\mathrm{Mg} 30$, hierarchical hybrid micro/nanostructured titanium surfaces followed by magnesium ion implantation for 30 minutes; Mg60, hierarchical hybrid micro/ nanostructured titanium surfaces followed by magnesium ion implantation for 60 minutes.

\section{Cell morphology and proliferation on titanium surfaces}

The actin cytoskeleton was labeled to observe cell morphology at 24 hours after seeding on the three titanium surfaces. The cells maintained their typical spindle morphology, while actin filaments showed improved spread on the magnesium-treated titanium surface, especially in the Mg60 group (Figure S3).

Cell proliferation on the specimens during the first 4 days of incubation was assessed using an MTT assay and the results are shown in Figure 3. There were significant differences between the three groups at days 1 and 4 . The cell metabolic activity on the magnesium-treated titanium surface was significantly greater than that of the control group, and
60 minutes of magnesium treatment showed the greatest cellular metabolic activity overall.

\section{Osteogenic differentiation activity in rBMMSCs on titanium surfaces}

The alkaline phosphatase staining results showed more pronounced alkaline phosphatase-positive areas on the magnesium-implanted micro/nanostructured titanium surfaces than on the controls (Figure 4A). The pronounced alkaline phosphatase-positive areas on the Mg60 surface were shown to be the best out of the three groups. Similar results were demonstrated by semiquantification of alkaline phosphatase (Figure 4C). 


\section{MTT results}

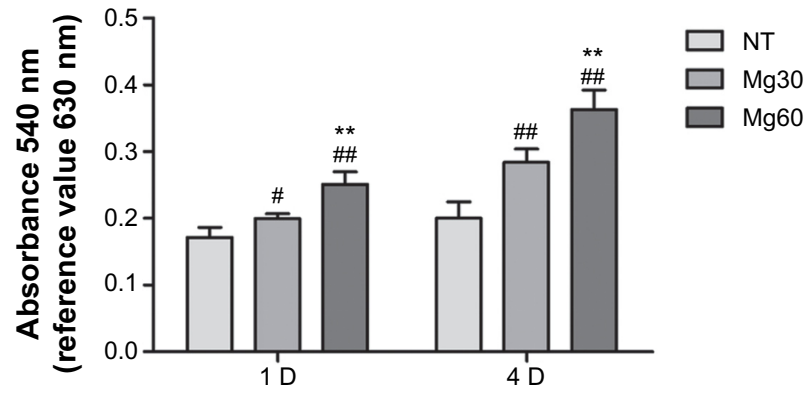

Figure 3 Analysis of cell metabolism on titanium substrates.

Notes: ${ }^{\#} P<0.05,{ }^{\#} P<0.01$ versus NT group; ${ }^{* * P}<0.01$ versus $M g 30$ group.

Abbreviations: MTT, methyl thiazolyl tetrazolium; NT, hierarchical hybrid micro/ nanostructured titanium surfaces; $\mathrm{Mg} 30$, hierarchical hybrid micro/nanostructured titanium surfaces followed by magnesium ion implantation for 30 minutes; $\mathbf{M g 6 0}$, hierarchical hybrid micro/nanostructured titanium surfaces followed by magnesium ion implantation for 60 minutes; $\mathrm{D}$, day.

Several osteogenic differentiation-related genes, including the early marker $A L P$ and the relatively late stage markers $O C N$ and $O P N$, were detected by the real-time polymerase chain reaction assay. All of these mRNAs were upregulated after 14 days of culture on the magnesium-implanted micro/ nanostructured titanium surfaces. The cells cultured on the Mg60 titanium surface showed the highest expression of both the early marker $A L P$ and the relatively late stage markers $O C N$ and $O P N$ (Figure 4B). The Western blot results for expression of osteogenic-related proteins in the rBMMSCs on the titanium specimens after 7 days of incubation are shown in Figure 4D. The levels of OCN, OPN, and alkaline phosphatase were increased in the Mg30 and Mg60 groups (with a larger increase in the Mg60 group), compared with expression of $\beta$-actin.

\section{Effects of extracts on rBMMSCs}

To understand the influence of the magnesium ions released from the bioactive ion implantation-treated micro/ nanostructured titanium surfaces, rBMMSCs were incubated in the extracts and subjected to cell proliferation and differentiation assays. As shown in Figure 5, the total cell metabolic activity increased during the observation period; however, there were no statistically significant differences between the three samples. Figure 6B shows that the osteogenic differentiation-related genes $O C N, O P N$, and $A L P$ were upregulated after 14 days of incubation with the collected extracts from magnesium-implanted micro/nanostructures. The Mg60 extraction showed the highest gene expression levels in comparison with Mg30 and NT extractions. Alkaline phosphatase staining and semiquantification assays further demonstrated the osteogenic differentiation ability of magnesium-containing extractions (Figure 6A and C).

Accumulation of magnesium ions released from the different titanium surfaces after 6 and 12 days of incubation in the
A
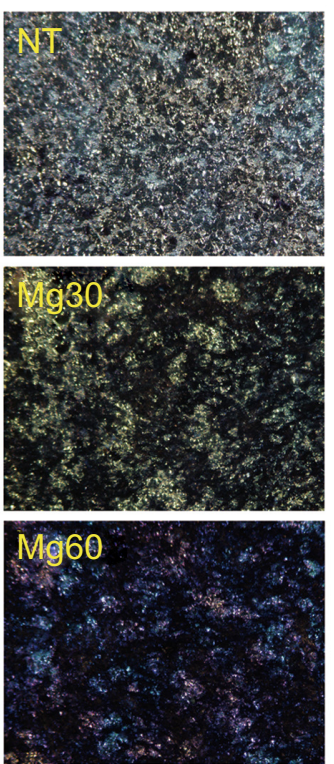

B
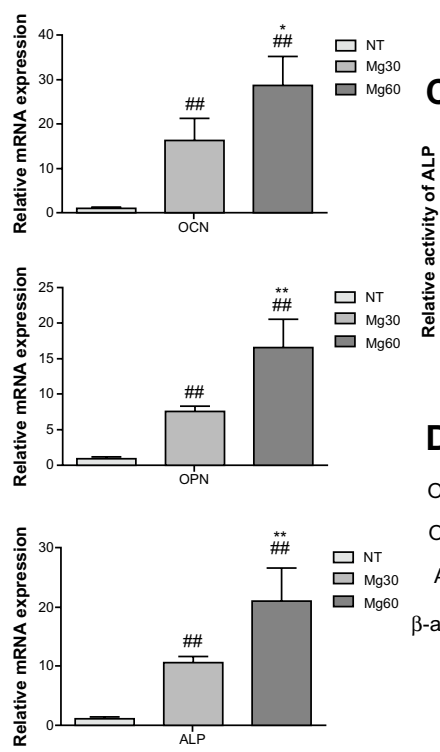

C

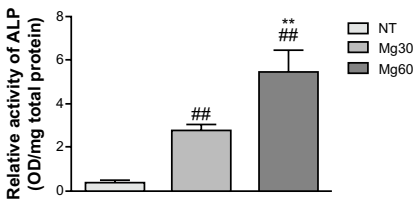

D

NT $\quad M g 30 \quad M g 60$

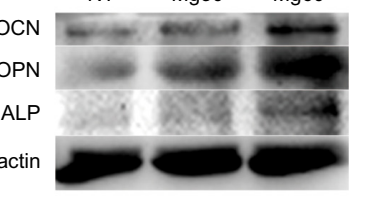

Figure 4 Analysis of cell differentiation on titanium substrates. Alkaline phosphatase staining $(\mathbf{A})$ and a quantitative assay (C) were carried out on the titanium surfaces. Expression levels of osteogenic-related differentiation genes and proteins (OCN, OPN, and alkaline phosphatase) were measured by real-time polymerase chain reaction and Western blot, respectively (B and $\mathbf{D})$.

Notes: ${ }^{\# P} P 0.01$ versus NT group; $* P<0.05, * * P<0.01$ versus $\mathrm{Mg} 30$ group.

Abbreviations: NT, hierarchical hybrid micro/nanostructured titanium surfaces; Mg30, hierarchical hybrid micro/nanostructured titanium surfaces followed by magnesium ion implantation for 30 minutes; Mg60, hierarchical hybrid micro/nanostructured titanium surfaces followed by magnesium ion implantation for 60 minutes; OD, optical density; OCN, osteocalcin; OPN, osteopontin; ALP, alkaline phosphatase. 
MTT results

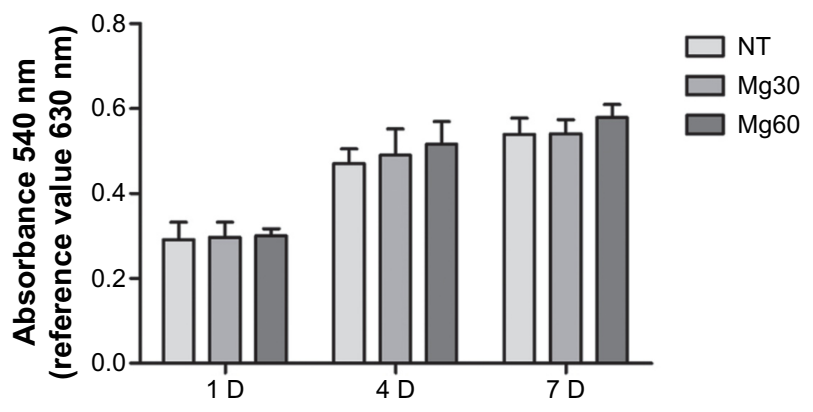

Figure $\mathbf{5}$ Analysis of cell metabolism in medium extractions.

Abbreviations: MTT, methyl thiazolyl tetrazolium; NT, hierarchical hybrid micro/ nanostructured titanium surfaces; $\mathrm{Mg} 30$, hierarchical hybrid micro/nanostructured titanium surfaces followed by magnesium ion implantation for 30 minutes; $\mathrm{Mg60}$, hierarchical hybrid micro/nanostructured titanium surfaces followed by magnesium ion implantation for 60 minutes; $\mathrm{D}$, day.

cell culture medium is shown in Figure 6D. Magnesium ions were detected in the extracts of both magnesium-implanted titanium surfaces throughout the observation period, and the amount of magnesium ions detected in the Mg60 extracts was higher than that in the $\mathrm{Mg} 30$ extracts at each time point.

\section{Discussion}

Both the topography and chemical characteristics of a material play a critical role in the overall bioactivity of an implant and in mediating cellular function. In a previous study, an NT-fabricated titanium surface was shown to have better cellular function than a microrough titanium surface treated by acid etching alone. ${ }^{13}$ In the present study, an Mg PIII technique was used to further improve the bioactivity and osteogenic activity of NT.

\section{Magnesium ion implantation}

PIII is a non-line-of-sight process that has been used for surface modification of various biomaterials. ${ }^{39}$ It is a material modification process by which different amounts of ions can be injected onto a surface. ${ }^{40,41}$ In this study, we successfully implanted different concentrations of magnesium ions onto the NT titanium surface using this technique. Large amounts of magnesium were released from both Mg30 and Mg60 within the first 6 days. Subsequently, lower concentrations of ions were released from the specimens. At each time point, the concentration of magnesium released from $\mathrm{Mg} 60$ was approximately twice that of $\mathrm{Mg30}$, which further confirms the ability of this technique to control ion concentration with high accuracy.

\section{Effect of magnesium ions on cell differentiation}

We next evaluated the effectiveness of the two magnesiumincorporating titanium surfaces on the osteogenesis of rBMMSCs. When cells were cultured on the different titanium surfaces, both magnesium-treated samples showed enhanced proliferation as well as upregulated alkaline phosphatase activity and osteogenic differentiation-related gene expression (including $O C N, O P N$, and $A L P$ ) compared with
A

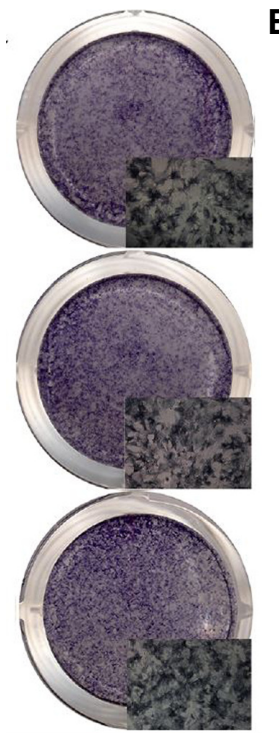

B

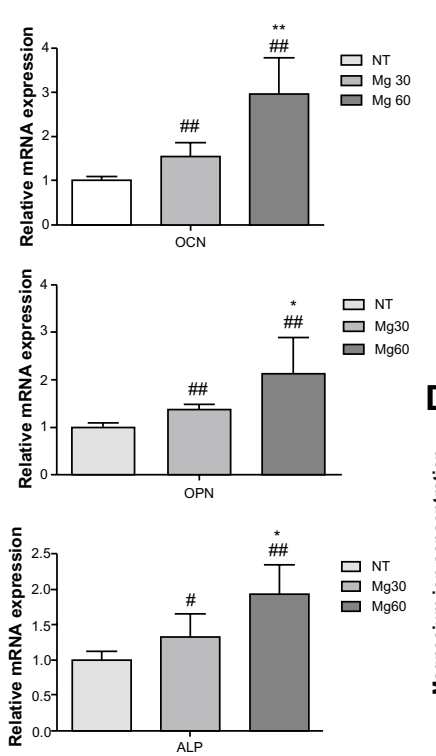

C

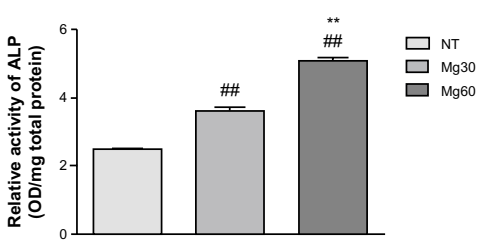

D

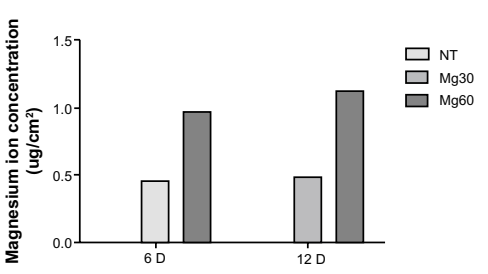

Figure 6 Analysis of cell differentiation in medium extractions and accumulated magnesium ion release. Alkaline phosphatase staining (A) and quantitative assay (C) carried out in medium extraction, real-time polymerase chain reaction assay in medium extractions (B), and accumulated magnesium ion release at 6 and 12 days (D).

Notes: ${ }^{*} P<0.05,{ }^{\# P}<0.01$ versus NT group; $* P<0.05$, $* * P<0.01$ versus $\mathrm{Mg} 30$ group.

Abbreviations: NT, hierarchical hybrid micro/nanostructured titanium surfaces; $\mathrm{Mg} 30$, hierarchical hybrid micro/nanostructured titanium surfaces followed by magnesium ion implantation for 30 minutes; Mg60, hierarchical hybrid micro/nanostructured titanium surfaces followed by magnesium ion implantation for 60 minutes; mRNA, messenger RNA; D, day; OCN, osteocalcin; OPN, osteopontin; ALP, alkaline phosphatase; OD, optical density. 
the control sample (NT). Enhanced cell responses were correlated with increasing concentration of magnesium ions. When cells were cultured in extract medium from the different specimens, alkaline phosphatase activity and osteogenic differentiation-related gene expression were also upregulated in a concentration-dependent manner in magnesium-modified titanium extractions compared with NT extractions. This clearly indicates the positive effects of magnesium ions on the osteogenic differentiation of rBMMSCs. Considering that a small amount $(50 \mu \mathrm{g} / \mathrm{mL})$ of magnesium ions can enhance osteogenic differentiation, as reported previously, ${ }^{42}$ our present data demonstrate that a lower concentration of magnesium (approximately $0.5 \mu \mathrm{g} / \mathrm{mL}$ ) could produce a similar effect.

\section{Effect of magnesium ions on cell proliferation}

Proliferation was significantly enhanced compared with controls when rBMMSCs were directly seeded on the two magnesium-treated titanium substrates. However, when cells were cultured in the different extraction media, there was no significant difference in cell proliferation between the groups. There are several factors that could have contributed to this finding.

First, alteration of surface micro/nanostructure patterns caused by Mg PIII may have caused this phenomenon; however, it is generally considered that the implant damage caused by this violent process can be reversed by postimplantation annealing without significant dimensional changes taking place during the process. ${ }^{43}$ There are also studies showing that the surface morphology becomes slightly smoother or damaged after ion implantation. ${ }^{28,44}$ In our present study, when Mg PIII was used for the first time to treat NT-modified titanium surfaces, a new intertwined micro/nanosheet surface appeared. With prolonged processing time, the altered intertwined micro/nanosheet became stronger. Therefore, gradual changes occurring on the micro/ nanostructures might have contributed to the accelerated cell proliferation when cells were cultured on the different substrates.

Second, when cells initially came into contact with the titanium surfaces, there was a distinct local microenvironment at the biomaterial interface, which was possibly different from other parts of the culture environment and, consequently, the collected extracts. Considering the ion diffusion kinetics, ${ }^{45,46}$ gradual dissolution of magnesium ions may have temporarily raised the local ionic concentration of biological fluids in the cell-biomaterial contact microenvironment. On the other hand, possible existence of the chemical form of this element may also play a positive role in the biological enhancement of rBMMSCs. We assume that these factors might lead to the accelerated cell proliferation on the plates rather than in the extracts. In addition, we cannot rule out the underlying combined effects of topography and chemical composition, including the physical and chemical forms of magnesium, in the cellbiomaterial contact microenvironment on cell proliferation ability. Regardless of the underlying mechanism, which needs further investigation, the final responses of cells on the magnesium-incorporated NT surfaces show clear benefits for potential bone regeneration and osseointegration.

\section{Conclusion}

In this study, magnesium-implanted titanium surfaces with a micro/nanostructure were prepared by acid etching and hydrothermal treatment followed by plasma immersion ion implantation to load different concentrations of magnesium. The magnesium-implanted micro/nanostructure-modified titanium surfaces facilitated the proliferation of rBMMSCs. Importantly, the osteogenic differentiation activity of these cells was promoted by the modified surface of their extractions. Magnesium-incorporated micro/nanostructured titanium surfaces are potential candidates for clinical applications to improve bone and titanium integration.

\section{Acknowledgments}

Joint financial support from the National Basic Research Program of China (973 Program, 2012CB933600), National Science Fund for Distinguished Young Scholars (81225006), Chang Jiang Scholars Program, National Natural Science Foundation of China (81271704, 31370962), and Shanghai Science and Technology R\&D Fund (11JC1413700, 13441902400) is acknowledged.

\section{Disclosure}

The authors report no conflicts of interest in this work.

\section{References}

1. Funk GF, Arcuri MR, Frodel JL Jr. Functional dental rehabilitation of massive palatomaxillary defects: cases requiring free tissue transfer and osseointegrated implants. Head Neck. 1998;20(1):38-51.

2. Anselme K. Osteoblast adhesion on biomaterials. Biomaterials. 2000;21(7):667-681.

3. Dohan Ehrenfest DM, Coelho PG, Kang BS, Sul YT, Albrektsson T. Classification of osseointegrated implant surfaces: materials, chemistry and topography. Trends Biotechnol. 2010;28(4):198-206.

4. Davies JE, Ajami E, Moineddin R, Mendes VC. The roles of different scale ranges of surface implant topography on the stability of the bone/ implant interface. Biomaterials. 2013;34(14):3535-3546. 
5. Takebe J, Ito S, Miura S, Miyata K, Ishibashi K. Physicochemical state of the nanotopographic surface of commercially pure titanium following anodization-hydrothermal treatment reveals significantly improved hydrophilicity and surface energy profiles. Mater Sci Eng C Mater Biol Appl. 2012;32(1):55-60.

6. SulYT, Johansson C, Wennerberg A, Cho LR, Chang BS, Albrektsson T. Optimum surface properties of oxidized implants for reinforcement of osseointegration: surface chemistry, oxide thickness, porosity, roughness, and crystal structure. Int J Oral Maxillofac Implants. 2005;20(3): 349-359.

7. Gittens RA, McLachlan T, Olivares-Navarrete R, et al. The effects of combined micron-/submicron-scale surface roughness and nanoscale features on cell proliferation and differentiation. Biomaterials. 2011;32(13):3395-3403.

8. Kieswetter K, Schwartz Z, Hummert TW, et al. Surface roughness modulates the local production of growth factors and cytokines by osteoblast-like MG-63 cells. J Biomed Mater Res. 1996;32(1):55-63.

9. Raines AL, Olivares-Navarrete R, Wieland M, et al. Regulation of angiogenesis during osseointegration by titanium surface microstructure and energy. Biomaterials. 2010;31(18):4909-4917.

10. Hansson S, Norton M. The relation between surface roughness and interfacial shear strength for bone-anchored implants. A mathematical model. J Biomech. 1999;32(8):829-836.

11. Boyan BD, Lossdörfer S, Wang L, et al. Osteoblasts generate an osteogenic microenvironment when grown on surfaces with rough microtopographies. Eur Cell Mater. 2003;6:22-27.

12. Zhang W, Wang G, Liu Y, et al. The synergistic effect of hierarchical micro/nano-topography and bioactive ions for enhanced osseointegration. Biomaterials. 2013;34(13):3184-3195.

13. Li J, Zhang W, Qiao Y, et al. Chemically regulated bioactive ion delivery platform on a titanium surface for sustained controlled release. $J$ Mater Chem B. 2014;2:283-294.

14. Sawada R, Kono K, Isama K, Haishima Y, Matsuoka A. Calciumincorporated titanium surfaces influence the osteogenic differentiation of human mesenchymal stem cells. J Biomed Mater Res A. 2013;101(9): 2573-2585.

15. Hass JL, Garrison EM, Wicher SA, et al. Synthetic osteogenic extracellular matrix formed by coated silicon dioxide nanosprings. J Nanobiotechnology. 2012;10:6.

16. Yu J, Li K, Zheng X, He D, Ye X, Wang M. In vitro and in vivo evaluation of zinc-modified Ca-Si-based ceramic coating for bone implants. PLoS One. 2013;8(3):e57564.

17. Park JW, Kim HK, Kim YJ, Jang JH, Song H, Hanawa T. Osteoblast response and osseointegration of a Ti-6Al-4V alloy implant incorporating strontium. Acta Biomater. 2010;6(7):2843-2851.

18. Shi X, Nakagawa M, Kawachi G, Xu L, Ishikawa K. Surface modification of titanium by hydrothermal treatment in $\mathrm{Mg}$-containing solution and early osteoblast responses. J Mater Sci Mater Med. 2012;23(5):1281-1290.

19. Boanini E, Gazzano M, Bigi A. Ionic substitutions in calcium phosphates synthesized at low temperature. Acta Biomater. 2010;6(6): 1882-1894.

20. Castellani C, Lindtner RA, Hausbrandt P, et al. Bone-implant interface strength and osseointegration: biodegradable magnesium alloy versus standard titanium control. Acta Biomater. 2011;7(1):432-440.

21. Hoppe A, Güldal NS, Boccaccini AR. A review of the biological response to ionic dissolution products from bioactive glasses and glass-ceramics. Biomaterials. 2011;32(11):2757-2774.

22. Boskey AL, Rimnac CM, Bansal M, Federman M, Lian J, Boyan BD. Effect of short-term hypomagnesemia on the chemical and mechanical properties of rat bone. J Orthop Res. 1992;10(6):774-783.

23. Zreiqat $\mathrm{H}$, Howlett CR, Zannettino A, et al. Mechanisms of magnesiumstimulated adhesion of osteoblastic cells to commonly used orthopaedic implants. J Biomed Mater Res. 2002;62(2):175-184.

24. Yamasaki Y, Yoshida Y, Okazaki M, et al. Synthesis of functionally graded $\mathrm{MgCO}_{3}$ apatite accelerating osteoblast adhesion. J Biomed Mater Res. 2002;62(1):99-105.
25. SulYT, Byon E, Wennerberg A. Surface characteristics of electrochemically oxidized implants and acid-etched implants: surface chemistry, morphology, pore configurations, oxide thickness, crystal structure, and roughness. Int J Oral Maxillofac Implants. 2008;23(4):631-640.

26. Cho LR, Kim DG, Kim JH, Byon ES, Jeong YS, Park CJ. Bone response of $\mathrm{Mg}$ ion-implanted clinical implants with the plasma source ion implantation method. Clin Oral Implants Res. 2010;21(8):848-856.

27. Kim BS, Kim JS, Park YM, Choi BY, Lee J. Mg ion implantation on SLAtreated titanium surface and its effects on the behavior of mesenchymal stem cell. Mater Sci Eng C Mater Biol Appl. 2013;33(3): 1554-1560.

28. Wong HM, Zhao Y, Tam V, et al. In vivo stimulation of bone formation by aluminum and oxygen plasma surface-modified magnesium implants. Biomaterials. 2013;34(38):9863-9876.

29. Jiang $X$, Zhao J, Wang $S$, et al. Mandibular repair in rats with premineralized silk scaffolds and BMP-2-modified bMSCs. Biomaterials. 2009;30(27):4522-4532.

30. Xia L, Zhang Z, Chen L, et al. Proliferation and osteogenic differentiation of human periodontal ligament cells on akermanite and B-TCP bioceramics. Eur Cell Mater. 2011;22:68-82.

31. Zhang W, Li Z, Liu Y, et al. Biofunctionalization of a titanium surface with a nano-sawtooth structure regulates the behavior of rat bone marrow mesenchymal stem cells. Int J Nanomedicine. 2012;7:4459-4472.

32. Wang S, Zhang Z, Zhao J, et al. Vertical alveolar ridge augmentation with beta-tricalcium phosphate and autologous osteoblasts in canine mandible. Biomaterials. 2009;30(13):2489-2498.

33. Kaur G, Wang C, Sun J, Wang Q. The synergistic effects of multivalent ligand display and nanotopography on osteogenic differentiation of rat bone marrow stem cells. Biomaterials. 2010;31(22):5813-5824.

34. Zou D, Han W, You S, et al. In vitro study of enhanced osteogenesis induced by HIF-1 $\alpha$-transduced bone marrow stem cells. Cell Prolif. 2011;44(3):234-243.

35. Zeng D, Xia L, Zhang W, et al. Maxillary sinus floor elevation using a tissue-engineered bone with calcium-magnesium phosphate cement and bone marrow stromal cells in rabbits. Tissue Eng Part A. 2012;18(7-8): $870-881$.

36. Shen Q, Zeng D, Zhou Y, et al. Curculigoside promotes osteogenic differentiation of bone marrow stromal cells from ovariectomized rats. $J$ Pharm Pharmacol. 2013;65(7):1005-1013.

37. Zhang W, Li Z, Huang Q, et al. Effects of a hybrid micro/nanorod topography-modified titanium implant on adhesion and osteogenic differentiation in rat bone marrow mesenchymal stem cells. Int $J$ Nanomedicine. 2013;8:257-265.

38. Zhang W, Wang X, Wang S, et al. The use of injectable sonicationinduced silk hydrogel for VEGF(165) and BMP-2 delivery for elevation of the maxillary sinus floor. Biomaterials. 2011;32(35):9415-9424.

39. Treves C, Martinesi M, Stio M, Gutiérrez A, Jiménez JA, López MF. In vitro biocompatibility evaluation of surface-modified titanium alloys. J Biomed Mater Res A. 2010;92(4):1623-1634.

40. Jin G, Cao H, Qiao Y, Meng F, Zhu H, Liu X. Osteogenic activity and antibacterial effect of zinc ion implanted titanium. Colloids Surf B Biointerfaces. 2014;117C:158-165.

41. Yang CH, Wang YT, Tsai WF, Ai CF, Lin MC, Huang HH. Effect of oxygen plasma immersion ion implantation treatment on corrosion resistance and cell adhesion of titanium surface. Clin Oral Implants Res. 2011;22(12):1426-1432.

42. Wong HM, Wu S, Chu PK, et al. Low-modulus Mg/PCL hybrid bone substitute for osteoporotic fracture fixation. Biomaterials. 2013;34(29): 7016-7032.

43. Tapash R, Rautray R, Narayanan R, Kim KH. Ion implantation of titanium based biomaterials. Prog Mater Sci. 2011;56(8):1137-1177.

44. Zheng Y, Li J, Liu X, Sun J. Antimicrobial and osteogenic effect of Agimplanted titanium with a nanostructured surface. Int J Nanomedicine. 2012;7:875-884.

45. Browne M, Gregson PJ. Effect of mechanical surface pretreatment on metal ion release. Biomaterials. 2000;21(4):385-392.

46. Wu X, Li J, Wang L, Huang D, Zuo Y, Li Y. The release properties of silver ions from Ag-nHA/TiO2/PA66 antimicrobial composite scaffolds. Biomed Mater. 2010;5(4):044105. 


\section{Supplementary materials}

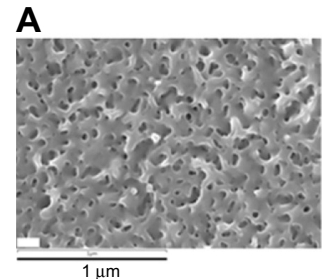

B

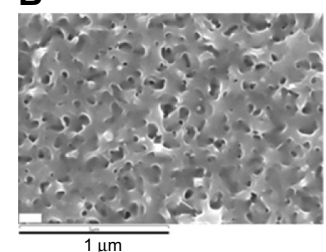

Mg Ko1_2

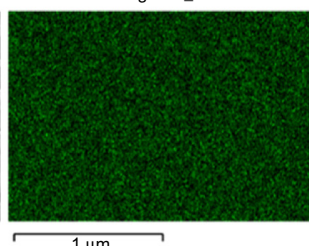

Mg Ka1_2

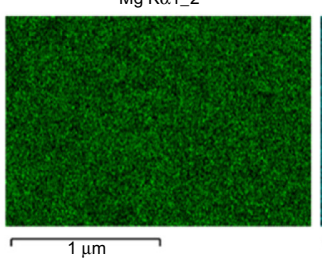

TiL $\alpha 1 \_2$

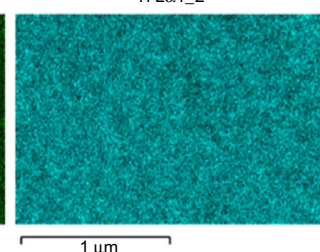

Ti L $\alpha 1 \_2$

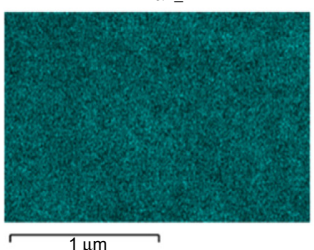

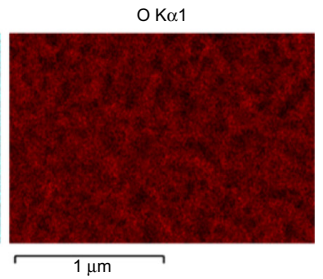

O K $\alpha 1$

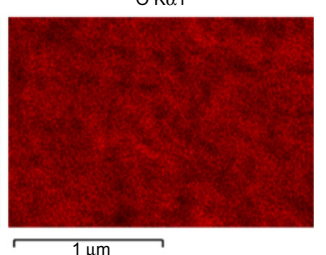

Figure SI Energy-dispersive X-ray spectrometric mapping results for two samples incorporating magnesium. Upper panel shows Mg30. Lower panel shows Mg60. 

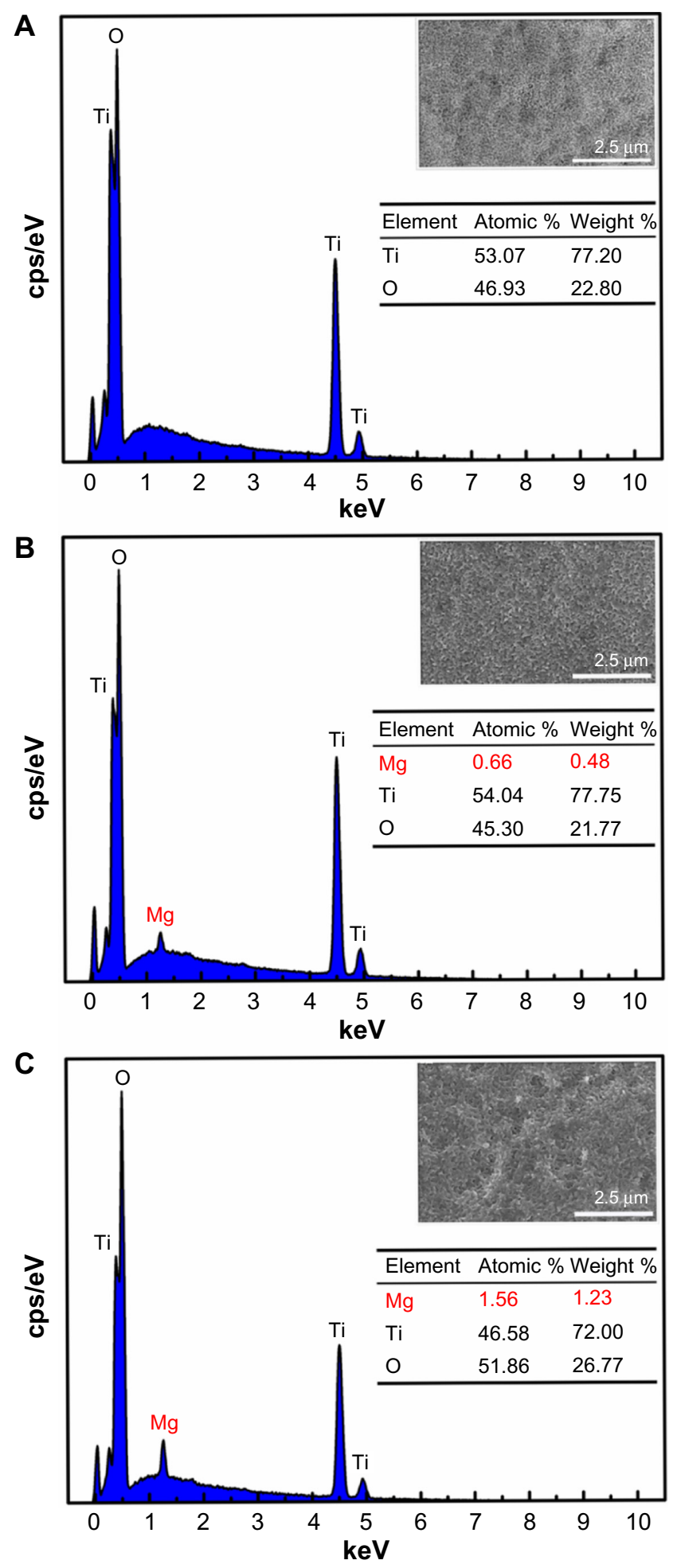

Figure S2 Energy-dispersive X-ray spectra obtained from NT (A), $\operatorname{Mg} 30$ (B) and $M g 60$ (C). These results are different from those shown in Figure 2, which is attributed to the difference in sampling depth between the scanning and transmission electron microscopy techniques. In more detail, scanning electron microscopy was used to determine the chemical composition of both the surface thin film and the underlying titanium substrate. As a result, the percent content of elemental magnesium was decreased by the larger metallic titanium content with only 0.66 at atomic \% for Mg30 and I.56 at atomic \% for Mg60. 

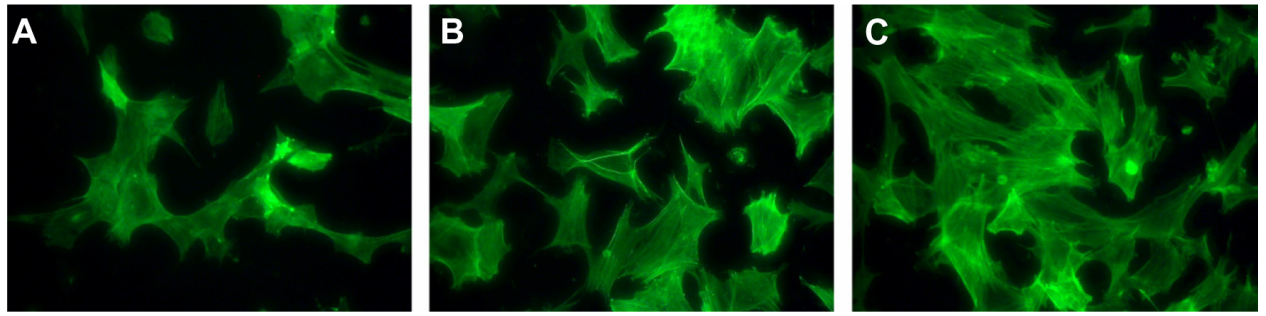

Figure S3 Morphology of cells on the titanium surfaces. Actin cytoskeletons were labeled to observe cell morphology at 24 hours after seeding on NT (A), Mg30 (B), and $\operatorname{Mg} 60(\mathbf{C})$.

Abbreviations: NT, hierarchical hybrid micro/nanostructured titanium surfaces; Mg30, hierarchical hybrid micro/nanostructured titanium surfaces followed by magnesium ion implantation for 30 minutes; Mg60, hierarchical hybrid micro/nanostructured titanium surfaces followed by magnesium ion implantation for 60 minutes.

\section{Publish your work in this journal}

The International Journal of Nanomedicine is an international, peerreviewed journal focusing on the application of nanotechnology in diagnostics, therapeutics, and drug delivery systems throughout the biomedical field. This journal is indexed on PubMed Central, MedLine, CAS, SciSearch $\AA$, Current Contents ${ }^{\circledR} /$ Clinical Medicine,
Journal Citation Reports/Science Edition, EMBase, Scopus and the Elsevier Bibliographic databases. The manuscript management system is completely online and includes a very quick and fair peer-review system, which is all easy to use. Visit http://www.dovepress.com/ testimonials.php to read real quotes from published authors. 